Portland State University

PDXScholar

$5-21-2020$

\title{
The Detrimental Side Effects of Retinol : Beyond Beauty Products
}

Michelle W. Lee

Portland State University

Follow this and additional works at: https://pdxscholar.library.pdx.edu/honorstheses Let us know how access to this document benefits you.

\section{Recommended Citation}

Lee, Michelle W., "The Detrimental Side Effects of Retinol : Beyond Beauty Products" (2020). University Honors Theses. Paper 895.

https://doi.org/10.15760/honors.916

This Thesis is brought to you for free and open access. It has been accepted for inclusion in University Honors Theses by an authorized administrator of PDXScholar. Please contact us if we can make this document more accessible: pdxscholar@pdx.edu. 


\title{
THE DETRIMENTAL SIDE EFFECTS OF RETINOL : BEYOND BEAUTY PRODUCTS
}

\author{
by \\ MICHELLE WAIYAN LEE
}

An undergraduate honors thesis submitted in partial fulfillment of the requirements for the degree of
Bachelor of Science
in

University Honors

and

Science

Thesis Advisor

Dr. Kim Brown 


\section{The Detrimental Side Effects of Retinol : Beyond Beauty Products}

Michelle Waiyan Lee

\section{Introduction :}

In past years, the market has seen a rise in demand for anti-aging products all across the world, thanks to society's ever growing obsession with physical appearance. The beauty industry in particular has profited off this vanity and shows no signs of stopping anytime soon. Social media plays a large part in the spread of skincare regimens, as well as beauty influencers who promote their products [1]. There has been a growing obsession to stay youthful and with still no certified way of freezing age, many consumers turn to makeup and skincare for answers. Among the anti-aging products available, a key player is retinol.

Often, retinol is associated with its functionality in the treatment of acne. It has become an increasingly popular choice among skincare ingredients, with products containing as little as $0.02 \%$ concentration. Makeup lines such as Glossier carry moisturizer that contains retinyl palmitate, a retinol ester. Retinyl palmitate is a weak ester in relation to retinol, yet it is enough for the company to market their moisturizer as an antiaging product [2]. Specifically, Retinol is a substance derived from Vitamin A and used to combat uneven skin tone [3]. The history of this Vitamin A derivative traces all the way back to Ancient Egypt, where animal liver was used to treat night blindness. The first study evaluating retinoic acid to treat acne was published in 1943 , and by the 1950s, retinoic acid was in the early stages of being a possible treatment for patients suffering from acne breakouts [4]. Retinoic acid was replaced by retinol in the $1990 \mathrm{~s}$, as retinol was a milder form of the substance and caused less skin irritation. Initially, concentrations of $0.25 \%$ retinol were prescribed to patients, since it was safe enough to not cause extreme irritation yet remained effective [5]. Dermatologists recommend to start using retinol at age forty, but nowadays, the use of the substance has become more common among twenty year olds as it is known to help soften skin and prevent wrinkles.

Retinol is found in small concentrations among many over the counter topicals, and the highest concentration of retinol one can obtain without a prescription is $2 \%$ in the United States and $1 \%$ in Canada. When applied to the face as a topical treatment, retinol acts as an exfoliant and peels off outer skin layers making way for new skin. Within our skin, there are structures called retinoic acid receptors (RAR) and retinoid X receptors (RXR). When prescription retinol in the form of retin-A or retinoic acid is applied, retinol binds to the receptors to speed up skin cell production. This causes old cells to die faster and speed up the growth of new cells. Retinol also encourages collagen production so, essentially, it triggers your skin to act younger [6]. Retinoids are classified into two different categories, either natural or synthetic. Natural retinoids are retinol, retinyl palmitate, retinyl acetate, 
retinaldehyde, isotretinoin and alitretinoin [7]. The active form of retinol is known as tretinoin.

After age 20, human skin begins to lose about $1 \%$ of collagen each year [8]. This process is also catalyzed by other factors such as chronic stress, poor diet, and smoking. Along with age, males and females experience a decline in hormones. A study published in the Acta Dermato-Venereologica showed that women appear to be at greater risk of developing wrinkles with age in comparison to men, and the source of this was hypo-estrogenism. Throughout the study, data indicated that the risk of facial wrinkling increases significantly with each full term pregnancy. Additionally, Hypo-estrogenism, or estrogen deficiency during post menopause, is associated with decreases in skin collagen, and eventually, wrinkling [9]. Functionally, Retinol helps contribute to epidermal thickness and even out skin complexion, specifically in those suffering from photodamaged skin, large pores, and hyperpigmentation. It is the strongest form of retinoid available without a prescription.

Tretinoin, the active form of retinol, speeds up the release of collagen, and protects against collagenase. Collagenase is an enzyme that breaks down collagen and its activity is triggered by sunlight exposure [10]. Subsequently, when a certain area of skin is treated with retinol, the user's skin may become more sensitive to UV rays [11]. As topicals, retinol can be applied to the face as a cream for individuals suffering from dry skin, or as a serum for individuals who have oily skin.

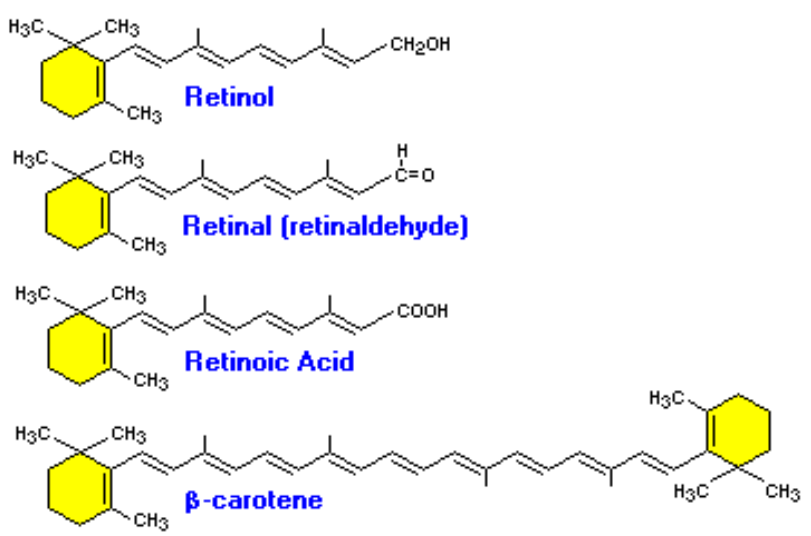

Figure 1: Chemical structures of Retinol, Retinaldehyde, Retinoic Acid, and Beta Carotene. All are precursors to Vitamin A [12].

\section{Research Question}

In this meta-analysis, the research question I am addressing is "What are the detrimental side effects of retinol?'. As consumers of beauty products get younger and younger, they are more susceptible to potential side effects due to their early initial ages starting the compounds and their prolonged use. Here I examine three potentially dangerous conditions (i.e., side effects) caused by misuse or overuse of retinol; pregnancy complications, infertility, and excessive skin peeling. Methodology used for assessment included a variety of review articles and studies on the topic as sources for this paper to further explore how retinol can have a potentially harmful effect on human physiology.

\section{Pregnancy Complications}

Aside from topical use via creams or moisturizers, retinol can be obtained from diet. Dietary retinol is especially important in pregnancy. The average daily intake for Vitamin A is 700 micrograms (mcg) retinol activity equivalent (RAE) 
[13]. During pregnancy, the average daily intake increases to $750 \mathrm{mcg}$, and during lactation it is 1,200 mcg RAE. One (1) International Unit (IU) retinol is equal to $0.3 \mathrm{mcg}$ RAE. The average daily dietary vitamin A intake in Americans above the age of two is 607 mcg RAE.

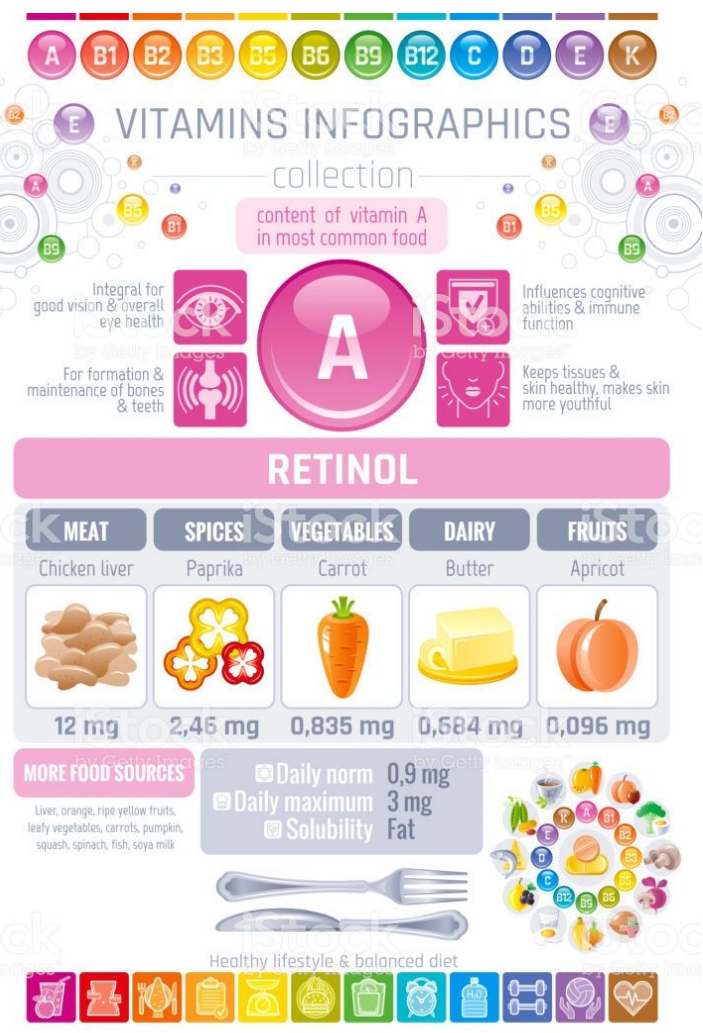

Figure 2 : Retinol can be obtained through diet, specifically Vitamin A rich foods [14].

Vitamin A is found in retinol, which means that it plays a part in glucose metabolism. It is involved in immune system function, vision, and maintenance of bodily organs. There are two forms of Vitamin A that can be consumed, either preformed Vitamin A, which includes retinol, or provitamin A carotenoids. Preformed Vitamin A is found in dairy products, fish, and meat. Milk and eggs also contain a certain amount of provitamin A carotenoids. Other foods that have Vitamin A are tomato juice, carrots, pistachios, and peppers. Once ingested into the body, retinol levels are tracked via plasma, or blood samples. Those who are at the highest risk of Vitamin A deficiency are pregnant women, individuals suffering from cystic fibrosis, and premature infants.

\subsection{Gestational Diabetes}

Gestational diabetes mellitus (GDM) is a condition in which pregnant women who have never had diabetes prior to pregnancy suddenly develop it. This is the most common during the second trimester of pregnancy and poses threats to fetal health [15]. A defining aspect of GDM is glucose intolerance. There are two types of GDM, with A1GDM being diet controlled gestational diabetes, and A2GDM being medical therapy [16]. Retinol binding protein 4 (RBP4) binds to retinol and is closely tied to insulin resistance [16]. During pregnancy, dieticians often advise women monitor Vitamin A intake as excessive levels can cause complications to both the mother and child. Preterm infants don't have adequate liver stores of Vitamin A at their time of birth, thus their plasma concentrations are low which can lead to eye and lung disease if not treated [11]. Without adequate amounts of Vitamin A in breast milk, infants aren't able to receive proper nutrients, which significantly impacts their growth. They also often develop night blindness as a result [17].

Gestational diabetes affects $1-14 \%$ of pregnancies across the world, and is higher than $10 \%$ in Asian countries. In a study done by Shenzhen Maternal and Child Health Hospital, 194 GDM patients were studied between 2014 to 2017 using blood samples collected during middle and late pregnancy stages. Results indicated RBP4 levels 
were highest during the lastest stages of pregnancy with a positive relationship observed between RBP4, insulin resistance and gestational diabetes. GDM mothers were also found to be at an increased risk of developing type 2 diabetes post pregnancy, given the increased levels of insulin resistance occurring during pregnancy. High levels of insulin resistance affect the body through an inability of the insulin receptor to be activated. This prevents the pancreas from producing enough insulin to stabilize blood sugar which can require medical intervention through self dosing by insulin injections.

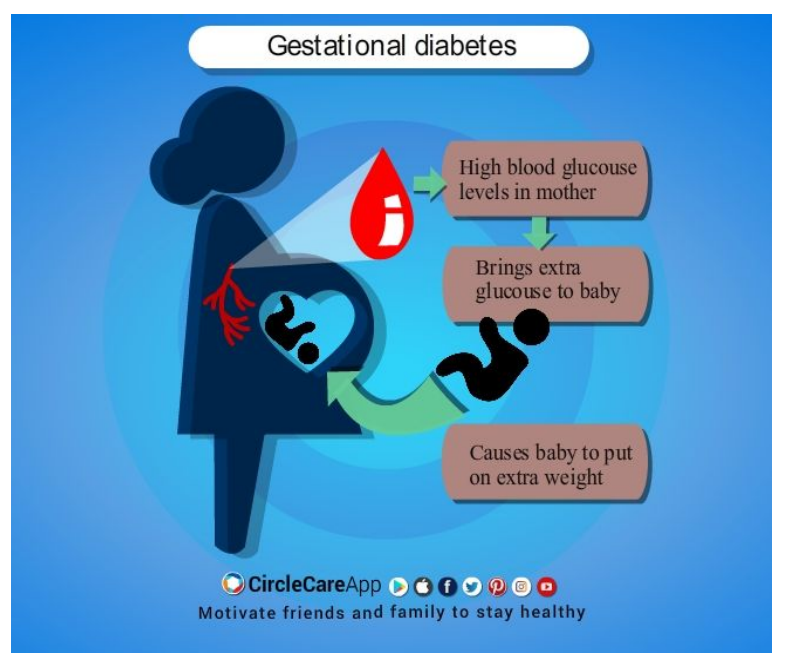

Figure 3 : Improper levels of Vitamin A during pregnancy can cause gestational diabetes [18].

\subsection{Maternal Anemia and Night Blindness}

A cross sectional study published in the Journal of Obstetrics and Gynecology explored how Vitamin A deficiency during pregnancy could lead to an increased risk of maternal anemia and delivery complications [15]. Anemia, or low red blood cell count, complicates pregnancies as there aren't enough red blood cells to facilitate the flow of oxygen from the mother to baby. This study examined 736 women during their third trimester of pregnancy and the factors used to measure health of the women included night blindness, hypertension levels, and gestational age of the infant. The women were from across all socioeconomic backgrounds, which was an important factor when considering accessibility to proper nutrients, specifically Vitamin A [17]. The women were split into two groups, and socioeconomic background was determined based highest level of education achieved, annual income, property holdings, and occupation. Results from this cross sectional study indicated that $2.9 \%$ of the women suffered from night blindness. Night blindness was tested via questionnaire, and it was concluded that all the women who suffered from night blindness were of low socioeconomic background. Thirty-five percent (35\%) of the women had low levels of retinol concentration and 41.2\% suffered from moderate to severe anemia. Fifteen point eight percent $(15.8 \%)$ of the women developed hypertension during the study. The authors also suggested that low levels of Vitamin A increased susceptibility to infection, thus low retinol concentrations trigger pregnancy anemia and contribute to other conditions such as night blindness and hypertension.

However, there are also dangers to excessive Vitamin A consumption. High Vitamin A during pregnancy equates to liver damage. Vitamin $\mathrm{A}$ is stored in the liver and is also fat soluble. When it accumulates in large amounts, it leads to liver damage that is irreversible. When retinol is used, it interacts with the hormone thyroxine and transthyretin. Thyroxine is produced in the thyroid gland and secreted into the bloodstream. Together, 
thyroxine and transthyretin form a complex that prevents the retinol binding protein from going to the kidney. Once in the bloodstream, thyroxine goes to the liver [19]. In addition, women who use topical isotretinoin exhibit an increased risk of birth defects in their children, specifically to the eyes and lungs. Retinol is currently under category $\mathrm{X}$ for pregnancy in the United States, meaning that studies have shown signs of fetal complications when pregnant women are exposed to retinol.

\subsection{Polycystic Ovarian Syndrome}

RBP4 is also thought to cause polycystic ovarian syndrome [20]. Polycystic ovarian syndrome (PCOS) is a hormonal syndrome that is common among women who are of reproductive age. The condition involves missed or excessive menstrual periods [21]. In women suffering from PCOS, the ovaries fail to release eggs. High RBP4 levels in women suffering from PCOS are thought to be influenced by androgen hormones. Androgen hormones, including testosterone, are more commonly associated as male hormones although they are produced in females as well, and high levels of androgens prevent the release of eggs [22]. When combined with estrogen, male hormones work to maintain proper bone mass and reproductive tissues in women. At age 19 and higher, the appropriate amount of testosterone in females falls between 8-60 nanograms per deciliter. Conversely in males, the average amount of testosterone is 240-950 nanograms per deciliter [23].

In stem cells, retinoid acid receptors aid in hematopoiesis, which is the formation of blood cellular structures present in bone marrow [24].
Retinoid signaling functions in helping to initiate meiosis of germ cells found in the fetal ovaries of mammals. Due to this, abnormal retinoid signaling is thought to be involved in the development of polycystic ovarian syndrome. Regulation of retinoid levels in the ovary allow for molecular control of ovarian development and oocyte maturation [25]. A less severe but common side effect of high androgen levels is acne. Women diagnosed with PCOS can lead to other fatal health conditions such as endometrial cancer and stroke [26]. Race and ethnicity do not impact an individual's risk of PCOS, but family history of obesity does. Women can prevent PCOS complications during pregnancy by maintaining a healthy blood sugar level before and throughout pregnancy, along with a healthy weight.

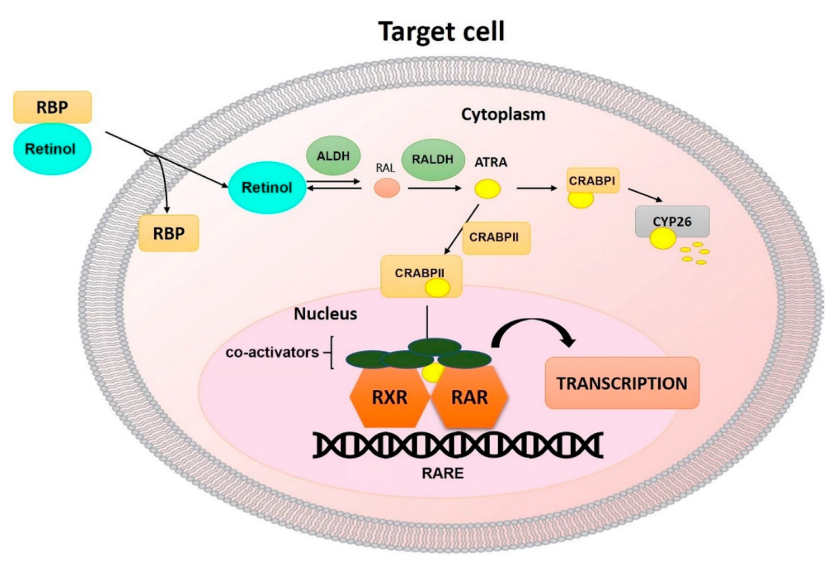

Figure 4: Regulation of Retinoid and RBP4 levels in cells is crucial to preventing Polycystic Ovarian Syndrome [27].

\section{Infertility}

Another ingredient found in retinol is Vitamin E. Not only is Vitamin E an antioxidant, but it is also one of the mechanisms by which the body 
protects itself from free radical damage. Without Vitamin E, there is an imbalance between the body's free radicals and antioxidants known as oxidative stress. A study done by the National Institute of Health and Environmental Protection of the Netherlands examined whether gender related differences in antioxidant status existed among couples who struggle with infertility. There are two types of infertility, primary and secondary. Primary infertility refers to couples who have not conceived after at least one year having intercourse without the use of birth control. Secondary infertility is when couples who have been able to get pregnant at least once are now unable to conceive again [28].

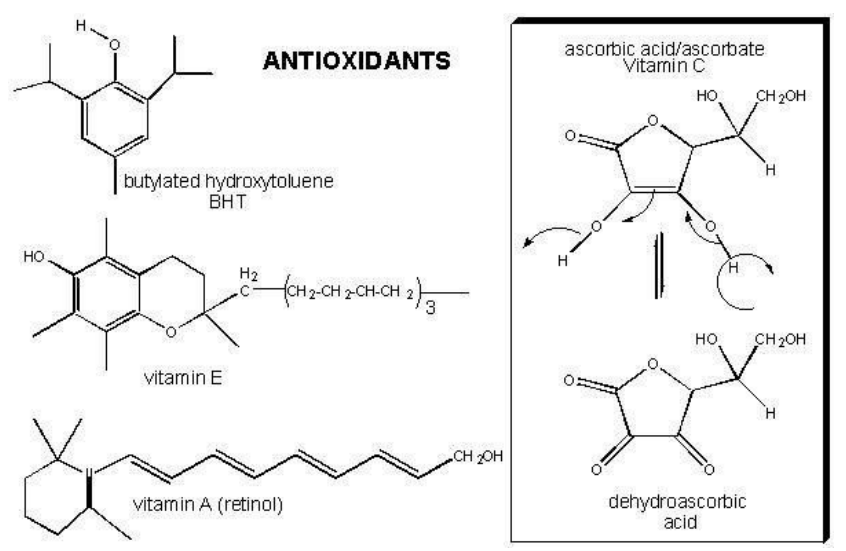

Figure 5: Vitamin $\mathrm{E}$ is an ingredient found in retinol and acts as an antioxidant. Antioxidants work to protect the body's cells from free radical damage [29].

In general, male infertility is often caused by cancer treatments, hormone imbalance, and is characterized by decreased sperm or blockage that prevents the sperm from being released. Antioxidants in the body help to reduce sperm DNA fragmentation which is essential to preventing mutagenic effects.
Patient treatment with oral antioxidant vitamins is a standard practice to improving male infertility [30]. Female infertility can be caused by diabetes, eating disorders, polycystic ovarian syndrome (PCOS), or thyroid disease. Smoking also has a large impact on male and female infertility.

A total of 40 couples were tested, and concentrations of retinol were measured using liquid chromatography after five days of sexual abstinence. In both men and women, FSH, LH and testosterone were measured after five days. For women, the hormones were measured on the fifth day of their menstrual cycles. From this study, it was found that infertility was influenced by age, body mass, and smoking habits. Men who had abnormal semen

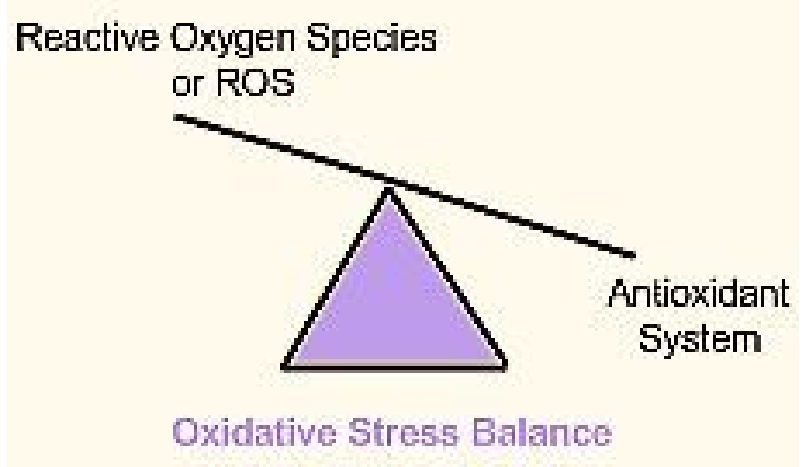

Figure 6: Oxidative stress is a condition caused by an imbalance between antioxidants and free radicals in the body [31].

concentrations had low levels of natural antioxidants, and women with low levels of natural antioxidants were found to suffer from anovulation, a condition in which the ovaries aren't able to release an oocyte. This results in no ovulation taking place [32]. More than $50 \%$ of the participants had a BMI of $30 \mathrm{~kg} / \mathrm{m} 2$, which is considered clinically obese, and $70 \%$ of the 
participants had primary infertility. A major finding in this study was that cigarette smoking played a large role in disrupting the balance and direction of oxidative stress. Heavy smoking was defined by smoking more than 20 cigarettes a day. Men who were heavy smokers had significantly reduced retinol concentrations compared to those who smoked less, or not at all. A total of 35\% male participants fell into the heavy smoking category, and none of the women participating were smokers.

\section{Excessive Skin Peeling}

Despite many beneficial effects, the topical application of retinoids often causes severe local irritation manifested as mild erythema and stratum corneum (outermost layer of skin) peeling of the skin. Many users of retinol experience temporary redness, flakiness and peeling, along with skin sensitivity. Side effects of retinol are known as retinization, or the adjustment period of skin. The inflammation is a primary response of skin to certain noxious agents [33]. The best way to handle peeling is to apply a moisturizer to the skin after retinol. The estimated amount of time for retinol to have its full effect is 4 to 5 weeks. If peeling and redness symptoms persist past the four to five week mark, this is usually indicative of sensitivity to retinol. Allergic reactions of retinol include hives, swelling of face, severe burning, blistering and changes in the color of treated skin [34].

Another explanation for these observations could be that some individuals' skin lack retinoid receptors, which are proteins that trigger collagen production. Excessive skin peeling can eventually lead to cracked skin and increases potential for sunburns [35]. Other factors that can worsen adverse reactions to retinol include dry air, wind, and hot water when showering. A study done on thirty healthy Caucasian females aged between 32 to 72 years found that individuals with sensitive skin were still able to use anti-aging products, as long as it was paired with a cleanser or moisturizer. In this study, aged skin was defined as "flattened and dry". It was also found that sensitive skin can also be influenced by hormones. The subjects used cleanser and moisturizer twice a day for four weeks. After every seven days, the skin of the subjects was measured with improvement in hydration and elasticity observed. Researchers determined it is best to use a concentration of $0.05 \%$ retinol for long term application, as it causes less irritation [7].

There are significant differences in facial skin surface lipids (SSL) between males and females. Skin lipids make up a large majority of the skin barrier. The barrier function of female skin is weaker than male skin, due to female skin having more unsaturated ceramides. Female skin is also softer than male skin because it contains higher amounts of glycerophosphate [36]. Another study conducted by the Journal of Dermatological Treatment found that when paired with a Cetaphil Dermacontrol Moisturizer, irritation by tretinoin became more mild [37]. The study involved a mix of male and female subjects over the age of 18 with healthy skin who applied topically $0.05 \%$ tretinoin cream to the whole face, and the Cetaphil Moisturizer on only one side. The purpose of this study was to determine whether a benefit to using moisturizing lotion with SPF 30 with retinol was present. Results indicated that $83 \%$ of the subjects experienced skin irritation on both sides of 
the face, but irritation was less severe on the side on which moisturizer had been applied [37].

Ceramides, which were found in the moisturizer, are the major lipid constituent of lamellar sheets present in the intercellular spaces of the stratum corneum. These lamellar sheets are thought to provide the barrier property of the epidermis [38]. Similar to collagen, natural ceramides produced by the body eventually decrease with age. Environmental factors such as weather and air pollution work against ceramides and make way for premature aging and skin thinning [39]. Thus, skincare products which incorporate ceramides can work to improve the skin barrier and increase hydration. Physicians typically prescribe the lowest concentration of retinol possible, and gradually increase concentration if needed, giving patients enough time for their skin to become tolerant of the substance. For children younger than 9 years old and suffering from acne, the use and dosage must be determined by a doctor [40].
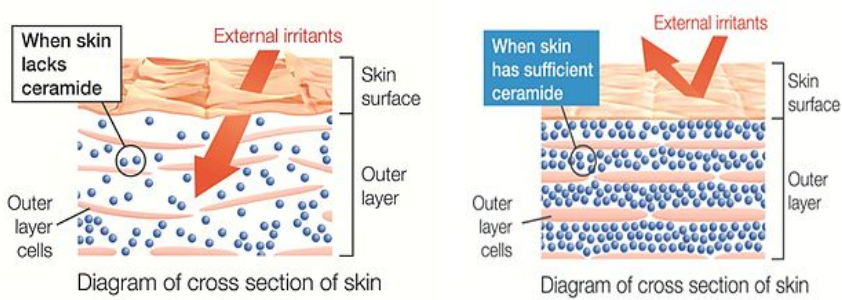

Figure 7 : Ceramides protect the skin against external irritants, thus proving to be an effective solution to reducing irritation [41].

The FDA has approved a retinoid called adapalene that contains $0.10 \%$ for anyone over the age of twelve [42]. However, retinoids sold in drugstores are less potent. For individuals suffering from acne, a skin condition that flares up during the time of adolescence, physicians recommend applying a pea sized amount to each problem area [43]. Teenagers may be prescribed retinol by their doctor if over the counter acne medications aren't working. Infants are known to have much more sensitive skin than adults. The stratum corneum functions to hold water and transport substances across the skin. This is also the outermost layer of skin where retinol is applied.

The Journal of the European Academy of Dermatology and Venereology states that although it may appear to be intact and fully functioning, infant skin does not finish developing until after the age of five [44]. This means that in the meantime, infants are susceptible to skin conditions such as eczema. There are multiple types of eczema, but the most common form is called atopic dermatitis. Atopic dermatitis is when the skin's natural barrier against the elements becomes weakened, leaving it susceptible to allergens. It is said that this condition can be attributed to genes, triggers in the environment, or immune system complications. Eczema is the most prominent during childhood and becomes mild during adult years. As a result, babies often develop rashes on their scalp and cheeks, and the skin becomes infected if scratched. Those suffering from eczema are advised not to use retinol because it can make these skin conditions worse [45]. 


\section{Conclusion :}

Despite these side effects, retinol is a relatively safe substance if used properly. Pregnancy complications associated with dietary retinol include development of gestational diabetes, maternal anemia, night blindness, and polycystic ovarian syndrome. Over-the-counter retinol undergoes a conversion process once inside the skin, before it can act on the receptors, which means it is less likely to irritate the skin than stronger prescription doses of retinol [6]. Retinoic Acid (also known as retin-A or tretinoin) is seriously potent and only available with a prescription. This is best for people who have been using over-the-counter (OTC) retinol consistently without a successful outcome and want something stronger, older complexions (40+) with deep set lines, or anyone with heavy pigmentation or acne. This is the most efficient form of vitamin $\mathrm{A}$ and acts immediately on receptors for fast and furious results. Both males and females will experience declines in hormones and collagen with age. However, in menopause, women are prone to a sudden estrogen drop, or hypo-estrogenism which leaves them more likely to develop skin wrinkling early on [8].

With tretinoin, it is advised to avoid using any other skin product that contains a peeling agent, such as benzoyl peroxide, or salicylic acid. Along with moisturizers, sunscreens with an SPF of 15 or higher can help [40]. There are potentially detrimental effects to excessive use of retinol whether it be topically or through diet. In this meta-analysis, I have conveyed the potentially harmful side effects of retinol, highlighting major categories of pregnancy complications, infertility, and excessive skin peeling. First time users of retinol should start in small concentrations beginning at $0.25 \%$ and increase over time if needed [5]. The general consensus is to start using retinol based products in your mid to late $20 \mathrm{~s}$, but it is also important to note that each individual's complexion ages at different rates and is influenced by various factors [6].

\section{Future Directions :}

It is important to consider individuals who have prior health conditions that might impact their bodily response to retinol consumption. This meta analysis drew from a variety of sources, but the information found is somewhat limited considering the short window of time given to construct this meta analysis. Studies in this meta analysis were published from different countries around the world, all with differing modes of healthcare and access to retinoids. If given more time, a topic to examine would be melanocytes and retinoid effectiveness across different ethnicities. Another subtopic of interest is botox and fillers. Specifically, collagen is used as an ingredient in dermal fillers. As agents of preventative aging, there have been numerous cases where such procedures have caused permanent damage to individuals. I would also like to examine the role of HGH (human growth hormone) in relation to collagen, since HGH helps with muscle development. 


\section{Literature Cited}

1. How Social Media is Shaping the Beauty Industry. (2019). Retrieved from https://influencermarketinghub.com/social-media-beauty-industry/

2. Villett, M. (2019, October 4). Reviewed: Glossier Priming Moisturizer (Will This Light Cream Give You Smooth, Hydrated Skin?). Retrieved from https://theskincareedit.com/2019/10/04/glossier-priming-moisturizer

3. Sadick, N. (2017). An Advanced, Physician Strength Retinol Peel Improves Signs of Aging and Acne Across a Range of Skin Types Including Melasma and Skin of Color . Journal of the American Academy of Dermatology, 76(6). doi: 10.1016/j.jaad.2017.04.157

4. The History of Retinol. (2020). Retrieved from https://www.pcaskin.com/the-history-of-retinol

5. How to Pick the Best Retinol Cream for You, According to a Dermatologist • Modern Dermatology Seattle Washington. (2019, July 16). Retrieved from https://mdinseattle.com/how-to-pick-the-best-retinol-cream-for-you-according-to-a-dermatologist/

6. Lawrenson, Amy. (2019, October 2). Retinol Rewind: What Type of Retinol is Best For Your Skin. Retrieved from https://edit.sundayriley.com/retinol-rewind-what-type-of-retinol-is-best-for-your-skin/

7. Babamiri, K., \& Nassab, R. (2010). Cosmeceuticals: The Evidence Behind the Retinoids. Aesthetic Surgery Journal, 30(1), 74-77. doi: 10.1177/1090820x09360704

8. Facko, Stacy. (2018). 6 Essential Compounds Your Body Stops Making As You Age. Retrieved from drclarkstore.com/blog/6-essential-compounds-your-body-stops-making-as-you-age/.

9. Youn, C. S., Kwon, O. S., Eun, H. C., Chung, J. H., Hwang, E. J., Park, B. J., \& Won, C. H. (2003). Effect of Pregnancy and Menopause on Facial Wrinkling in Women. Acta Dermato-Venereologica, 83(6), 419-424. doi: 10.1080/00015550310015464

10. Jiang, W. C., Zhang, H., Xu, Y., Jiang, C., Xu, Y., Liu, W., \& Tan, Y. (2019). Cutaneous vessel features of sensitive skin and its underlying functions. Skin Research and Technology. doi: 10.1111/srt.12819

11. When Beauty Products Cause Sun Sensitivity. (2019, August 15). Retrieved from https://www.skincancer.org/blog/when-beauty-products-cause-sun-sensitivity/

12. Bowen, R. (2017). [Vitamin A Retinol]. Retrieved from http://www.vivo.colostate.edu/hbooks/pathphys/topics/vitamina.html

13. Johnson, E. J. (2019, October 11). Office of Dietary Supplements - Vitamin A. Retrieved from https://ods.od.nih.gov/ 
14. (2018). [Retinol Vitamin A Food Icons]. Retrieved from https://www.istockphoto.com/vector/retinol-vitamin-a-food-icons-healthy-eating-flat-icon-set-text-letter-sy mbol-gm923274492-253438604

15. Gursoy, A. Y., Aynaoglu, G., Caglar, G. S., \& Soylemez, F. (2014). Early second trimester retinol-binding protein-4 values in cases with or without gestational diabetes mellitus risk factors: A cross-sectional study. Journal of Obstetrics and Gynaecology Research, 41(1), 55-61. doi: 10.1111/jog.12499

16. Du, X., Dong, Y., Xiao, L., Liu, G.-H., Qin, W., \& Yu, H. (2019, September). Association between retinol-binding protein 4 concentrations and gestational diabetes mellitus (A1GDM and A2GDM) in different pregnancy and postpartum periods. Retrieved from https://www.ncbi.nlm.nih.gov/pmc/articles/PMC6803236/

17. Radhika, M., Bhaskaram, P., Balakrishna, N., Ramalakshmi, B., Devi, S., \& Kumar, B. S. (2002). Effects of vitamin A deficiency during pregnancy on maternal and child health. BJOG: An International Journal of Obstetrics and Gynaecology, 109(6), 689-693. doi:10.1111/j.1471-0528.2002.01010.x

18. (2015). [What is the main cause of gestational diabetes]. Retrieved from http://www.mycirclecare.com/main-cause-gestational-diabetes/

19. Monaco, H. (2000, October 18). The Transthyretin-Retinol-Binding Protein Complex. Retrieved from https://www.researchgate.net/publication/12267651_The_Transthyretin-Retinol-Binding_Protein_Complex

20. Mellati, A. A., Sharifi, F., Sajadinejad, M., Sohrabi, D., \& Mazloomzadeh, S. (2011). The relationship between retinol-binding protein 4 levels, insulin resistance, androgen hormones and polycystic ovary syndrome. Scandinavian Journal of Clinical and Laboratory Investigation, 72(1), 39-44. doi: 10.3109/00365513.2011.626071

21. Watson, S. (2019, March 29). Polycystic Ovary Syndrome (PCOS): Symptoms, Causes, and Treatment. Retrieved from https://www.healthline.com/health/polycystic-ovary-disease

22. Fink, D., Polycystic Ovary Syndrome. (2019, April 1). Retrieved from https://www.womenshealth.gov/a-z-topics/polycystic-ovary-syndrome

23. Severson A., Barclay R., Bailey J. (2018). Testosterone Levels by Age: Normal Levels, Low T Signs . Retrieved from https://www.healthline.com/health/low-testosterone/testosterone-levels-by-age

24. Rosa et al. (2019, June 14). The Pleiotropic Role of Retinoic Acid/Retinoic Acid Receptors Signaling: From Vitamin A Metabolism to Gene Rearrangements in Acute Promyelocytic Leukemia. Retrieved from https://www.mdpi.com/1422-0067/20/12/2921/htm

25. Jiang, Y., Li, C., Chen, L., Wang, F., \& Zhou, X. (2017). Potential role of retinoids in ovarian physiology and pathogenesis of polycystic ovary syndrome. Clinica Chimica Acta, 469, 87-93. doi:

10.1016/j.cca.2017.03.025 
26. Polycystic Ovarian Syndrome. (2019). Retrived from

https://www.womenshealth.gov/a-z-topics/polycystic-ovary-syndrome

27. (2019). [Mechanisms of RA signaling]. Retrieved from https://www.mdpi.com/1422-0067/20/12/2921/htm

28. Infertility: MedlinePlus Medical Encyclopedia. (2020). Retrieved from https://medlineplus.gov/ency/article/001191.htm

29. (2010). [Well Known Antioxidants]. Retrieved from

https://www.researchgate.net/figure/Well-known-antioxidants_fig1_325455460

30. Ménézo, Y. J., Hazout, A., Panteix, G., Robert, F., Rollet, J., Cohen-Bacrie, P., .. Benkhalifa, M. (2007). Antioxidants to reduce sperm DNA fragmentation: an unexpected adverse effect. Reproductive BioMedicine Online, 14(4), 418-421. doi: 10.1016/s1472-6483(10)60887-5

31. (2009). [Oxidative Stress]. Retrieved from https://www.glutathione-for-health.com/oxidative-stress.html

32. Al-Azemi, M., Omu, A., Fatinikun, T., Mannazhath, N., \& Abraham, S. (2009). Factors contributing to gender differences in serum retinol and $\alpha$-tocopherol in infertile couples. Reproductive BioMedicine Online, 19(4), 583-590. doi: 10.1016/j.rbmo.2009.05.005

33. Kim, B. (2003). The mechanism of retinol-induced irritation and its application to anti-irritant development. Toxicology Letters, 146(1), 65-73. doi: 10.1016/j.toxlet.2003.09.001

34. Cunha, J. (2018, July 25). Side Effects of Retin-AMicro (Tretinoin Gel), Warnings, Uses. Retrieved from https://www.rxlist.com/retin-a-micro-side-effects-drug-center.htm\#consumer

35. Spada, F., Lui, A. H., \& Barnes, T. M. (2019). Use of formulations for sensitive skin improves the visible signs of aging, including wrinkle size and elasticity. Clinical, Cosmetic and Investigational Dermatology, Volume 12, 415-425. doi: 10.2147/ccid.s212240

36. Cui, L., He, C. F., Fan, L. N., \& Jia, Y. (2018). Application of lipidomics to reveal differences in facial skin surface lipids between males and females. Journal of Cosmetic Dermatology, 17(6), 1254-1261. doi: 10.1111 jocd. 12474

37. Matsunaga, K., Leow, Y.-H., Chan, R., Kerrouche, N., \& Paliargues, F. (2012). Adjunctive usage of a non-comedogenic moisturizer with adapalene gel $0.1 \%$ improves local tolerance: a randomized, investigator-blinded, split-face study in healthy Asian subjects. Journal of Dermatological Treatment, 24(4), 278-282. doi: 10.3109/09546634.2012.661037

38. Coderch, L., Lopez, O., Maza, A. D. L., \& Parra, J. L. (2003). Ceramides and Skin Function. American Journal of Clinical Dermatology, 4(2), 107-129. doi: 10.2165/00128071-200304020-00004

39. Teas, J. (2018, December 5). This Skin-Care Ingredient Is a Secret Fountain of Youth. Retrieved from 
https://www.thecut.com/2018/12/a-guide-to-ceramides-in-skin-care.html

40. Tretinoin (Topical Route) Precautions. (2020, February 1). Retrieved from https://www.mayoclinic.org/drugs-supplements/tretinoin-topical-route/precautions/drg-20066521?p=1

41. (1987). [Benefits of Ceramides]. Retrieved from

https:/gunillaofsweden.com/benefits-of-ceramides-in-skin-care/

42. Gohara, M. (2017, March 31). FDA Approves a Prescription-Strength Retinol for Over-the-Counter Use. Retrieved from https://www.fitnessmagazine.com/health/fda-approves-a-prescription-strength-retinol-for-over-the-counteruse/

43. Hyman, M. (2017, December 20). Skin Problems in Children. Retrieved from https://my.clevelandclinic.org/health/articles

44. Ribet, V., Gurdak, M., Ferret, P. J., Brinio, E., Labadie, F. G., \& Rossi, A. (2019). Stepwise approach of development of dermo-cosmetic products in healthy and atopic dermatitis paediatric population: safety evaluation, clinical development and postmarket surveillance. Journal of the European Academy of Dermatology and Venereology, 33(12), 2319-2326. doi: 10.1111/jdv.15785

45. 7 Types of Eczema: Symptoms, Causes, and Pictures. (2005). Retrieved from https://www.healthline.com/health/types-of-eczema 